\title{
The Effect of Variable Lateral Stress of Laminated Bamboo with Artificially Dent Surface on Mechanical Properties
}

\author{
Iskandar Yasin, Henricus Priyosulistyo, Suprapto Siswosukarto, Ashar Saputra
}

\begin{abstract}
Since many years ago bamboo has been used, in addition to wood, for structural components in rural areas due to easily growth and its short harvest time. The properties of laminated bamboo depend on many aspects, among others: quality of glue, lateral stresses during manufacture and roughness of bamboo lamina. This research used Bambusa Dendrocalamus Asper (local name: Bambu Petung) and aimed to determine the effect of artificial dented surface of the laminas on mechanical properties with variable lateral stresses. The dimensions and the types of the specimen were based on ISO 2004 standard. The age of bamboo specimens was 6 years and the lateral stresses were varied from $0 \mathrm{MPa}$ to $2.5 \mathrm{MPa}$ with $0.5 \mathrm{MPa}$ interval. Physical properties of the specimen such as water content (humidity), specific gravity, were performed prior to the mechanical tests (tensile, compression, shear and flexural tests). Analysis of results was performed by statistical method, in addition to scanning electron microscopy for further inspection of microscopic deterioration.
\end{abstract}

Keywords - Bamboo Lamina, Lateral Stress, Statistical Method, SEM

\section{Background}

At this time the timber with good quality has been difficult to obtain, so the wood is increasingly rare for building construction. However, the wood will be needed because it has several advantages over the baton and steel. The advantages of wood of which have good mechanical properties, wherein the weight ratio of the power compared to a higher kind than concrete and steel in addition to approaching the renewable nature and aesthetics.

Iskandar Yasin

Universitas Sarjanawiyata Tamansiswa

Indonesia

Henricus Priyosulistyo

Universitas Gadjah Mada

Indonesia

Suprapto Siswosukarto

Universitas Gadjah Mada

Indonesia

Ashar Saputra

Universitas Gadjah Mada

Indonesia
Bamboo grows fast, easy to grow and does not require special maintenance. Bamboo with good quality can be harvested at the age ranges (3-5) years [1]. Bamboo with good quality can be harvested at the age ranges (3-4) years [2]. The advantages of laminated bamboo can be formed in a variety of sizes, better mechanical properties than the base material type of bamboo used [1].

Glue line damage to become laminated bamboo beams crack before its power reaches the maximum value. Bamboo blade in laminated bamboo beams are experiencing compression estimated damage to the fiber and therefore contribute to the strength and power of the glueline laminated bamboo beam.

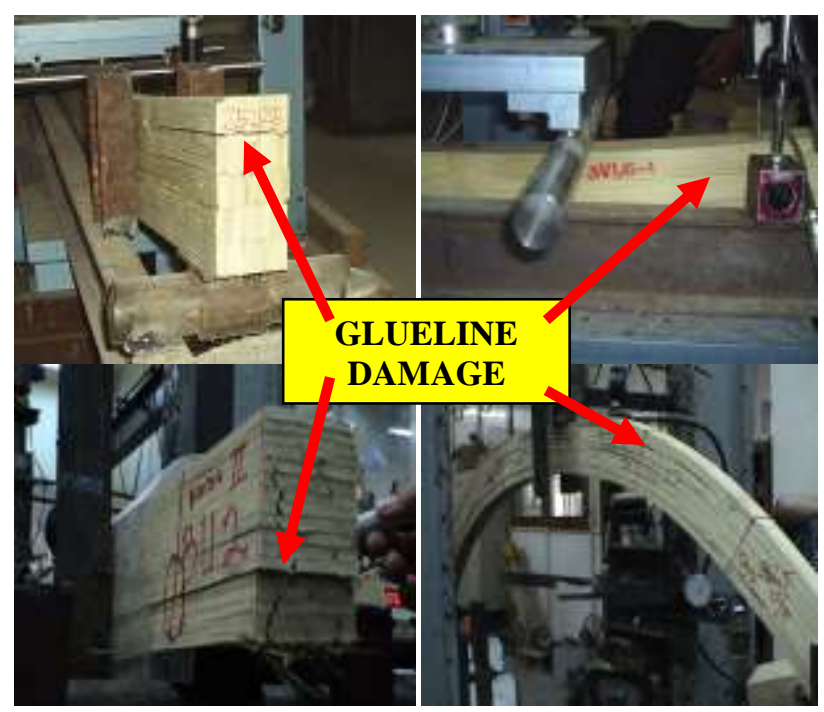

Figure 1. Bamboo Lamination Beam Glueline Damages

The study was conducted to determine the effect of pressure felt on physical and mechanical properties of bamboo. Pressure felts made on bamboo slats with pressure variations Felts $0 \mathrm{MPa}, 1.5 \mathrm{MPa}, 2 \mathrm{MPa}$ and $2.5 \mathrm{MPa}$.

\section{Literature Review}

The influence of lateral stress on the shear strength of the beam clamp horizontally laminated bamboo Petung has been investigated by Oka [3]. Masrizal [4], conducted research on the effect of compression force MoR Petung Bamboo 
Proc. of the Third Intl. Conf. on Advances in Civil and Structural Engineering - CSE 2015

Copyright (C) Institute of Research Engineers and Doctors, USA .All rights reserved.

ISBN: 978-1-63248-057-6 doi: 10.15224/ 978-1-63248-057-6-48

laminated beams vertically. MOR maximum value obtained in advance of $1.3 \mathrm{MPa}$ compression force $\mathrm{s} / \mathrm{d} 1.6 \mathrm{MPa}$. Likewise, the influence of pressure on the shear strength of the beam clamp horizontally laminated bamboo Ampel has been investigated by Amirullah [2].

Ochi [5], conducted research the effect of tensile strength of bamboo fiber bamboo laminate applications using biodegradable-plastic resin thereby increasing the tensile strength and bamboo reaches $70 \%$ of the tensile strength without resin.

\section{Research Method}

The raw material is Bambusa Dendrocalamus asper. Making the preliminary test specimen to test the properties of physics and mechanics were based on ISO 2004 Standard and ASTM D143-2008.

Experimental tests performed on specimens with lateral stress variations. Testing the physical properties consist of water content and density, while testing the mechanical properties of bamboo consists of flexural strength, tensile strength, shear strength and compressive strength.

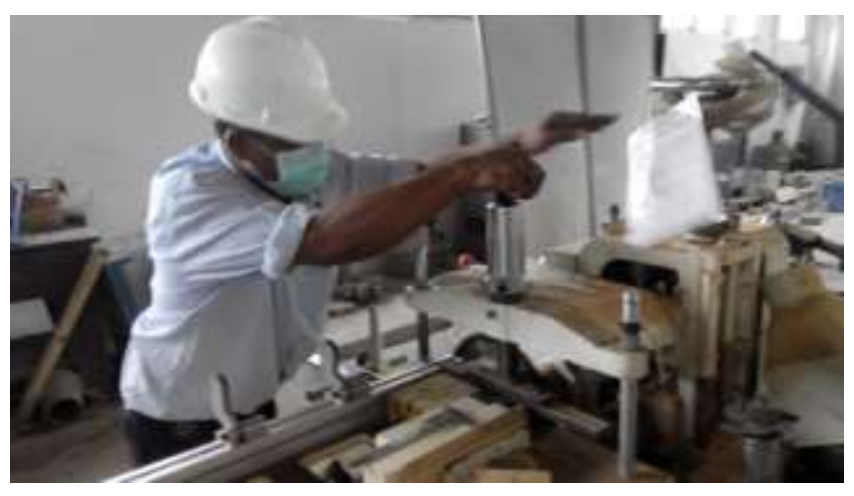

Figure 2. Specimen Manufacturing

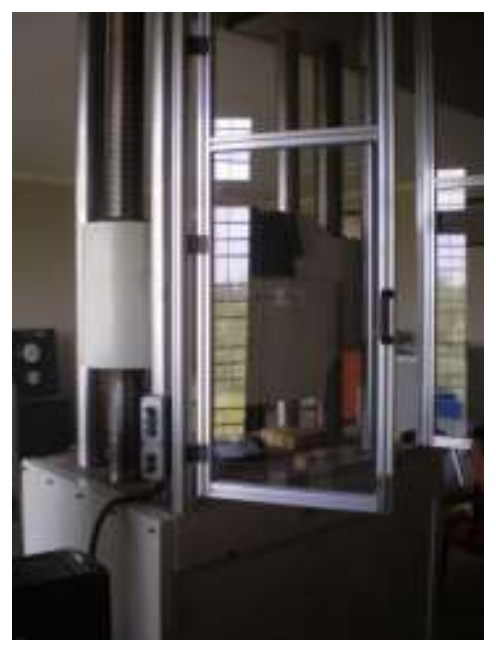

Figure 3. Universal Testing Machine
TABLE I. SPECIMEN QUANTITY

\begin{tabular}{|c|c|c|c|c|c|}
\hline \multirow{2}{*}{ No. } & \multirow{2}{*}{ Testing Type } & \multirow{2}{*}{$\begin{array}{c}\text { Without } \\
\text { Lateral Stress }\end{array}$} & \multicolumn{3}{|c|}{ Lateral Stress Variations } \\
\hline & & & $1.5 \mathrm{MPa}$ & $2.0 \mathrm{MPa}$ & $2.5 \mathrm{MPa}$ \\
\hline 1 & Water Content & 30 & 30 & 30 & 30 \\
\hline 2 & Specific Gravity & 30 & 30 & 30 & 30 \\
\hline 4 & Compression Strengtt & 30 & 30 & 30 & 30 \\
\hline 5 & Tensile Strength & 30 & 30 & 30 & 30 \\
\hline 6 & Shear Strength & 30 & 30 & 30 & 30 \\
\hline 7 & Flexural Strength & 30 & 30 & 30 & 30 \\
\hline & Total Quantities & 180 & 180 & 180 & 180 \\
\hline
\end{tabular}

\section{Iv. Result and Discussion}

The water content of lateral stress $0 \mathrm{MPa}$ on average $12.95 \%$. At a lateral stress $1.5 \mathrm{MPa}$ the water content is an average of $12.81 \%$. The water content of lateral stress $2 \mathrm{MPa}$ is an average of $12.52 \%$. And the water content of lateral stress $2.5 \mathrm{MPa}$ average water content of $12.43 \%$.

TABLE II. WATER CONTENT AND LATERAL STRESS PARTIAL CORRELATION

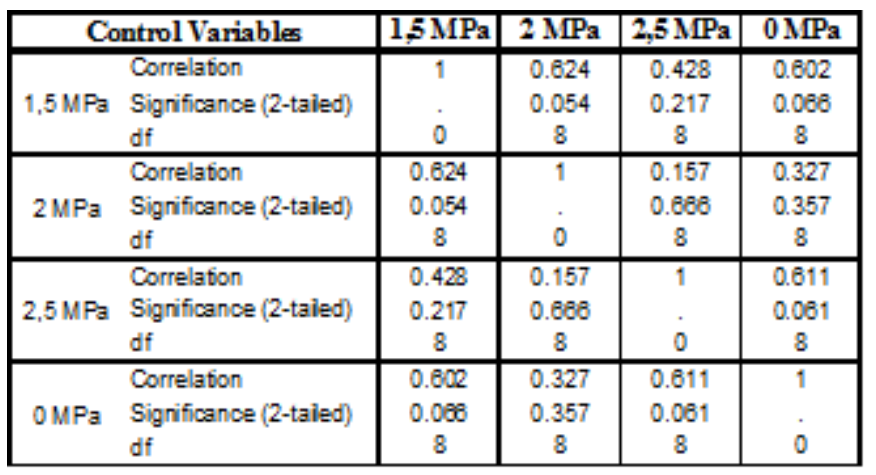

Specific gravity on lateral stress $0 \mathrm{MPa}$ an average of 0.64 $\mathrm{g} / \mathrm{cm} 3$. At lateral stress $1.5 \mathrm{MPa}$ the value was an average density of $0.73 \mathrm{~g} / \mathrm{cm} 3$. Density values with lateral stress 2 $\mathrm{MPa}$ is an average of $0.75 \mathrm{~g} / \mathrm{cm} 3$. At lateral stress $2.5 \mathrm{MPa}$ specific gravity value average of $0.78 \mathrm{~g} / \mathrm{cm} 3$.

TABLE III. SPECIFIC GRAVITY AND LATERAL STRESS PARTIAL CORRELATION

\begin{tabular}{|c|c|c|c|c|c|}
\hline \multicolumn{2}{|r|}{ Control Variables } & $1,5 \mathrm{MPa}$ & $2 \mathrm{MPa}$ & $2,51 \mathrm{MPa}$ & OTMPa \\
\hline $1,5 \mathrm{MPa}$ & $\begin{array}{l}\text { Correlation } \\
\text { Significance (2-talled) } \\
\text { of }\end{array}$ & 1 & $\begin{array}{c}0.165 \\
0.65 \\
8 \\
\end{array}$ & $\begin{array}{c}-0.193 \\
0.593 \\
8 \\
\end{array}$ & $\begin{array}{c}0.314 \\
0.376 \\
8 \\
\end{array}$ \\
\hline $2 \mathrm{MPa}$ & $\begin{array}{l}\text { Correlation } \\
\text { Significance }(2-\text { talled }) \\
\text { of }\end{array}$ & $\begin{array}{c}0.165 \\
0.65 \\
8\end{array}$ & $\begin{array}{l}1 \\
0\end{array}$ & $\begin{array}{c}0.221 \\
0.54 \\
8\end{array}$ & $\begin{array}{c}0.378 \\
0.282 \\
8\end{array}$ \\
\hline $2,5 \mathrm{MPa}$ & $\begin{array}{l}\text { Correlation } \\
\text { Significance (2-talled) } \\
\text { of }\end{array}$ & $\begin{array}{c}-0.193 \\
0.593 \\
8\end{array}$ & $\begin{array}{c}0.221 \\
0.54 \\
8\end{array}$ & 1 & $\begin{array}{c}0.236 \\
0.511 \\
8\end{array}$ \\
\hline $\mathrm{OMPa}$ & $\begin{array}{l}\text { Correlation } \\
\text { Significance }(2-\text { talled }) \\
\text { of }\end{array}$ & $\begin{array}{c}0.314 \\
0.376 \\
8\end{array}$ & $\begin{array}{c}0.378 \\
0.282 \\
8\end{array}$ & $\begin{array}{c}0.236 \\
0.511 \\
8\end{array}$ & 1 \\
\hline
\end{tabular}

Compressive strength bamboo with $0 \mathrm{MPa}$ Lateral stress were $45.80 \mathrm{MPa}$. At lateral stress $1.5 \mathrm{MPa}$ had a strong average 70.08. At lateral stress $2 \mathrm{MPa}$ the compressive strength were an average 54.65 MPa. Lateral stress at 2.5 MPa averaged 51.15 MPa. 
Proc. of the Third Intl. Conf. on Advances in Civil and Structural Engineering - CSE 2015

Copyright (C) Institute of Research Engineers and Doctors, USA .All rights reserved.

ISBN: 978-1-63248-057-6 doi: 10.15224/ 978-1-63248-057-6-48

TABLE IV. COMPRESSION STRENGTH AND LATERAL STRESS PARTIAL CORRELATION

\begin{tabular}{|c|c|c|c|c|c|}
\hline \multicolumn{2}{|c|}{ Control Variables } & $1,5 \mathrm{MPa}$ & $2 \mathrm{MPa}$ & $2,5 \mathrm{MPa}$ & $0 \mathrm{MPa}$ \\
\hline \multirow{3}{*}{$1,5 \mathrm{MPa}$} & Correlation & 1 & 0.115 & 0.12 & -0.285 \\
\hline & Significance (2-tailed) & . & 0.547 & 0.527 & 0.127 \\
\hline & $d f$ & 0 & 28 & 28 & 28 \\
\hline \multirow{3}{*}{$2 \mathrm{MPa}$} & Correlation & 0.115 & 1 & 0.082 & -0.218 \\
\hline & Significance (2-tailed) & 0.547 & & 0.685 & 0.248 \\
\hline & $d f$ & 28 & 0 & 28 & 28 \\
\hline \multirow{3}{*}{$2,5 \mathrm{MPa}$} & Correlation & 0.12 & 0.082 & $\overline{1}$ & 0.162 \\
\hline & Significance (2-tailed) & 0.527 & 0.685 & . & 0.391 \\
\hline & df & 28 & 28 & 0 & 28 \\
\hline \multirow{3}{*}{$O \mathrm{MPa}$} & Correlation & -0.285 & -0.218 & 0.162 & 1 \\
\hline & Significance (2-tailed) & 0.127 & 0.248 & 0.391 & \\
\hline & $d f$ & 28 & 28 & 28 & 0 \\
\hline
\end{tabular}

The average Flexural strength at lateral stress $0 \mathrm{MPa}$ was 132.54 MPa. Average flexural strength at lateral stress 1.5 $\mathrm{Mpa}$ is $146.69 \mathrm{MPa}$. Flexural strength with forged pressure of $2 \mathrm{MPa}$ is an average of $122.01 \mathrm{MPa}$. Flexural strength of bamboo with a pressure of $2.5 \mathrm{MPa}$ was forged by an average of $121.52 \mathrm{MPa}$.

TABlE V. FleXural Strength ANd LATERAL StRESS Partial CORRELATION

\begin{tabular}{|c|c|c|c|c|c|}
\hline \multicolumn{2}{|c|}{ Control Varlables } & $1,5 \mathrm{MPa}$ & $2 \mathrm{MPa}$ & $2,5 \mathrm{MPa}$ & $O \mathrm{MPa}$ \\
\hline $1,5 \mathrm{MPa}$ & $\begin{array}{l}\text { Correlation } \\
\text { Significance (2-taled) } \\
\text { df }\end{array}$ & $\begin{array}{l}1 \\
0\end{array}$ & $\begin{array}{c}-0.026 \\
0.894 \\
28\end{array}$ & $\begin{array}{c}0.019 \\
0.919 \\
28\end{array}$ & $\begin{array}{c}0.18 \\
0.34 \\
28\end{array}$ \\
\hline $2 \mathrm{MPa}$ & $\begin{array}{l}\text { Correlation } \\
\text { Significance (2-taled) } \\
\text { df }\end{array}$ & $\begin{array}{c}-0.026 \\
0.894 \\
28\end{array}$ & $\begin{array}{l}1 \\
0\end{array}$ & $\begin{array}{c}0.011 \\
0.953 \\
28\end{array}$ & $\begin{array}{c}0.02 \\
0.915 \\
28\end{array}$ \\
\hline $2,5 \mathrm{MPa}$ & $\begin{array}{l}\text { Correlation } \\
\text { Slgnificance (2-taled) } \\
\text { of }\end{array}$ & $\begin{array}{c}0.019 \\
0.919 \\
28\end{array}$ & $\begin{array}{c}0.011 \\
0.953 \\
28\end{array}$ & $\begin{array}{l}1 \\
0\end{array}$ & $\begin{array}{c}0.289 \\
0.122 \\
28\end{array}$ \\
\hline OMPa & $\begin{array}{l}\text { Correlation } \\
\text { Significance (2-taled) } \\
\text { df }\end{array}$ & $\begin{array}{c}0.18 \\
0.34 \\
28\end{array}$ & $\begin{array}{c}0.02 \\
0.915 \\
28\end{array}$ & $\begin{array}{c}0.289 \\
0.122 \\
28\end{array}$ & 1 \\
\hline
\end{tabular}

The average of tensile strength with lateral stress $0 \mathrm{MPa}$ is 192.74 MPa. Average tensile strength with lateral stress 1.5 $\mathrm{MPa}$ is $216.37 \mathrm{MPa}$. Tensile strength of bamboo with lateral stress $2 \mathrm{MPa}$ is the average of $164.75 \mathrm{MPa}$. At lateral stress 2.5 MPa tensile strength of bamboo is average 160.74 MPa.

TABLE VI. Tensile StREnGth AND LATERAL StRESS Partial CORRELATION

\begin{tabular}{|c|c|c|c|c|c|}
\hline & Control Variables & $1,5 \mathrm{MPa}$ & $2 \mathrm{MPa}$ & $2,5 \mathrm{MPa}$ & $0 \mathrm{MPa}$ \\
\hline 1,5 MPa & $\begin{array}{l}\text { Correlation } \\
\text { Significance (2-tailed) } \\
\text { df }\end{array}$ & $\begin{array}{l}1 \\
0\end{array}$ & $\begin{array}{c}-0.149 \\
0.432 \\
28\end{array}$ & $\begin{array}{c}-0.106 \\
0.579 \\
28\end{array}$ & $\begin{array}{c}0.003 \\
0.987 \\
28\end{array}$ \\
\hline $2 \mathrm{MPa}$ & $\begin{array}{l}\text { Correlation } \\
\text { Significance (2-tailed) } \\
\text { df }\end{array}$ & $\begin{array}{c}-0.149 \\
0.432 \\
28\end{array}$ & $\begin{array}{l}1 \\
0\end{array}$ & $\begin{array}{c}0.079 \\
0.678 \\
28\end{array}$ & $\begin{array}{c}-0.491 \\
0.006 \\
28\end{array}$ \\
\hline $2,5 \mathrm{MPa}$ & $\begin{array}{l}\text { Correlation } \\
\text { Significance (2-tailed) } \\
\text { df }\end{array}$ & $\begin{array}{c}-0.106 \\
0.579 \\
28\end{array}$ & $\begin{array}{c}0.079 \\
0.678 \\
28\end{array}$ & $\begin{array}{l}1 \\
0 \\
0\end{array}$ & $\begin{array}{c}-0.26 \\
0.166 \\
28\end{array}$ \\
\hline $0 \mathrm{MPa}$ & $\begin{array}{l}\text { Correlation } \\
\text { Significance (2-tailed) } \\
\text { df }\end{array}$ & $\begin{array}{c}0.003 \\
0.987 \\
28\end{array}$ & $\begin{array}{c}-0.491 \\
0.006 \\
28\end{array}$ & $\begin{array}{c}-0.26 \\
0.166 \\
28\end{array}$ & $\begin{array}{l}\overline{1} \\
0\end{array}$ \\
\hline
\end{tabular}

Shear strength of bamboo with lateral stress $0 \mathrm{MPa}$ pressure is an average of $25.41 \mathrm{MPa}$. At lateral stress $1.5 \mathrm{MPa}$ an average of $22.05 \mathrm{MPa}$. Shear strength of bamboo with lateral stress $2 \mathrm{MPa}$ is an average of $17.12 \mathrm{MPa}$. While the shear strength of bamboo were lateral stress of $2.5 \mathrm{MPa}$ is the average $15.05 \mathrm{MPa}$.

TABLE VII. SHEAR STRENGTH AND LATERAL STRESS PARTIAL CORRELATION

\begin{tabular}{|c|c|c|c|c|}
\hline Control Variables & $1,5 \mathrm{MPa}$ & $2 \mathrm{MPa}$ & $2,5 \mathrm{MPa}$ & $0 \mathrm{MPa}$ \\
\hline Correlation & 1 & 0.004 & 0.295 & -0.115 \\
$1,5 \mathrm{Mpg}$ Significan 2 (2-tailed) &. & 0.985 & 0.114 & 0.545 \\
df & 0 & 28 & 28 & 28 \\
\hline Correlation & 0.004 & 1 & -0.137 & 0.088 \\
$2 \mathrm{MPa}$ Significance (2-tailed) & 0.985 &. & 0.471 & 0.645 \\
df & 28 & 0 & 28 & 28 \\
\hline Correlation & 0.295 & -0.137 & 1 & 0.202 \\
$2,5 \mathrm{MPa}$ Significance (2-tailed) & 0.114 & 0.471 &. & 0.285 \\
df & 28 & 28 & 0 & 28 \\
\hline Correlation & -0.115 & 0.088 & 0.202 & 1 \\
\hline $\mathrm{MPg}$ Significance (2-tailed) & 0.545 & 0.645 & 0.285 &. \\
df & 28 & 28 & 28 & 0 \\
\hline
\end{tabular}

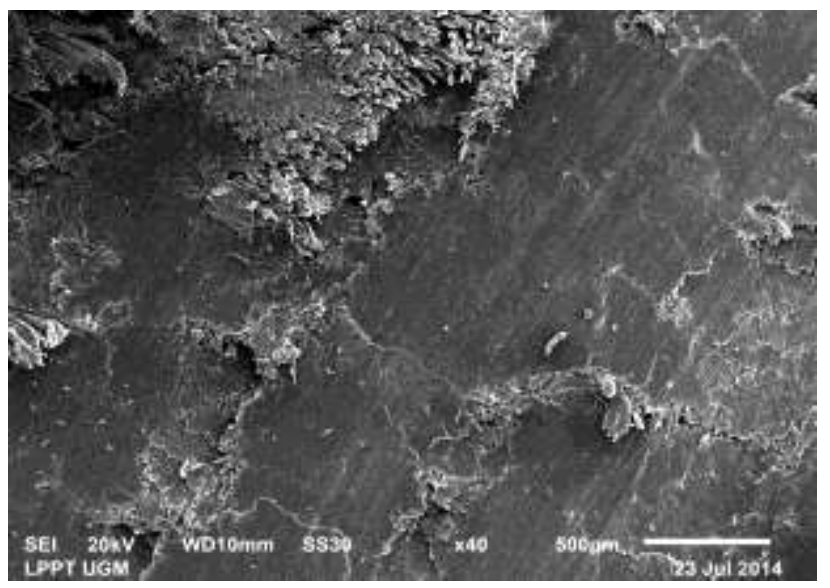

Figure 3. Specimen Without Lateral Stresses

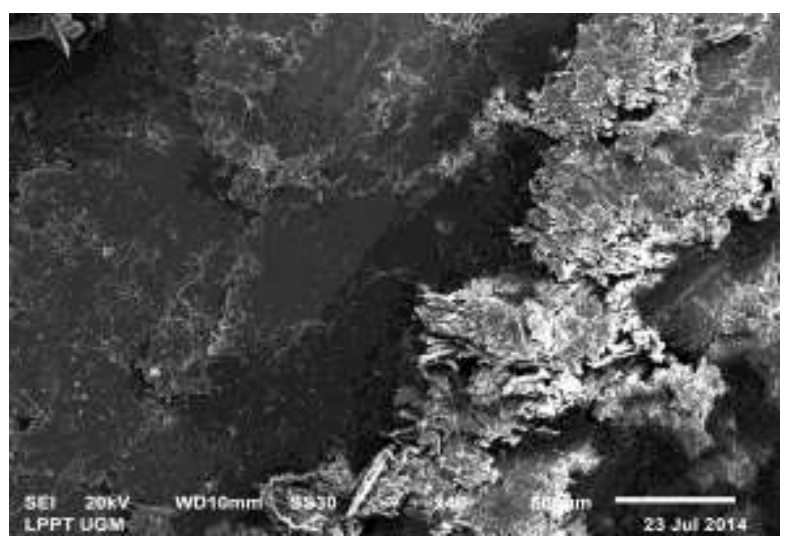

Figure 4. Specimen With Lateral Stresses 1,5 MPa 


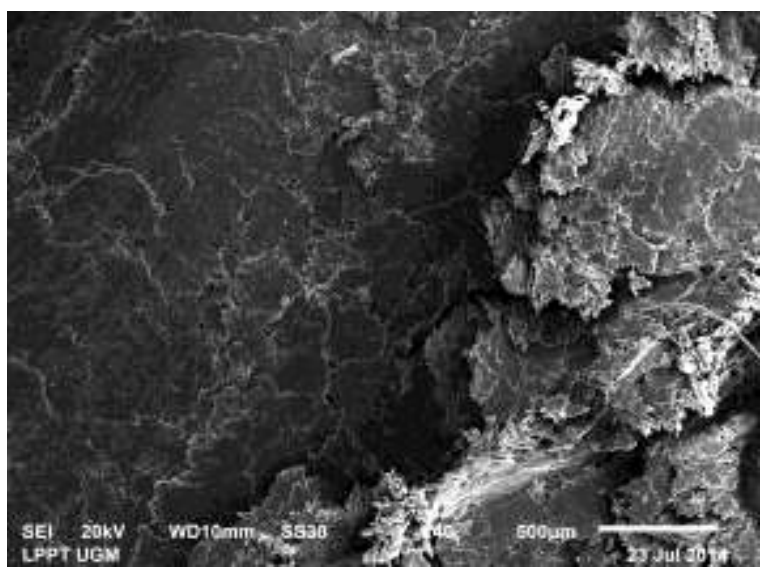

Figure 5. Specimen With Lateral Stresses $2 \mathrm{MPa}$

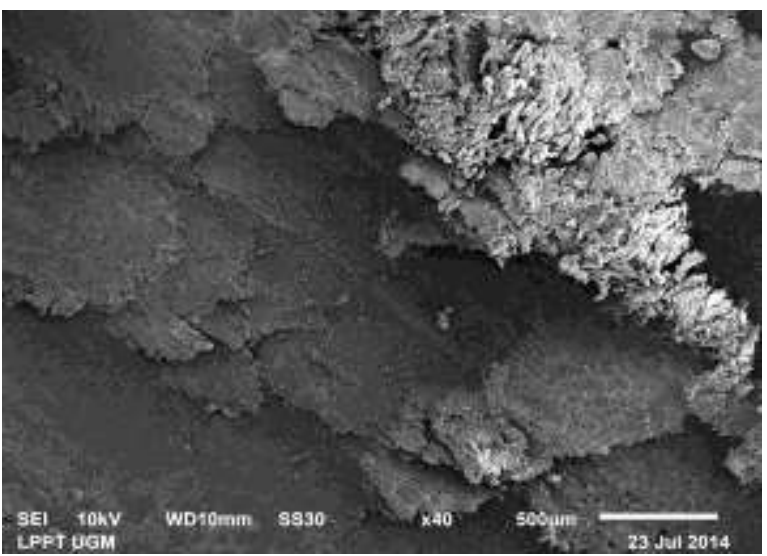

Figure 6. Specimen With Lateral Stresses 2,5 MPa

\section{v. Conclusion}

Average of bamboo water content with lateral stress of 0 $\mathrm{MPa}, 1.5 \mathrm{MPa}, 2 \mathrm{MPa}$ and $2.5 \mathrm{MPa}$ were $12.95 \%, 12.81 \%$, $12.52 \%$, and $12,43 \%$. The Average of bamboo specific gravity with lateral stress of $0 \mathrm{MPa}, 1.5 \mathrm{MPa}, 2 \mathrm{MPa}$ and 2.5 $\mathrm{MPa}$ were $0.64 \mathrm{~g} / \mathrm{cm} 3,0.73 \mathrm{~g} / \mathrm{cm} 3,0.75 \mathrm{~g} / \mathrm{cm} 3$ and $0.78 \mathrm{~g}$ $/ \mathrm{cm} 3$. Statistical analysis shows the physical properties of bamboo Petung not significantly with lateral stress.

Average flexural strength of bamboo with lateral stress of $0 \mathrm{MPa}, 1.5 \mathrm{MPa}, 2 \mathrm{MPa}$ and $2.5 \mathrm{MPa}$ were $132.54 \mathrm{MPa}$, 146.69 $\mathrm{MPa}, 122.01$ and $121 \mathrm{MPa}, 52 \mathrm{MPa}$. Average shear strength of bamboo with lateral stress of $0 \mathrm{MPa}, 1.5 \mathrm{MPa}, 2$ $\mathrm{MPa}$ and 2.5 $\mathrm{MPa}$ were 25.41 $\mathrm{MPa}, 22.05 \mathrm{MPa}, 17.12 \mathrm{MPa}$ and $15.05 \mathrm{MPa}$. Average compressive strength of bamboo with lateral stress of $0 \mathrm{MPa}, 1.5 \mathrm{MPa}, 2 \mathrm{MPa}$ and $2.5 \mathrm{MPa}$ were $45.79 \mathrm{MPa}, 70.08 \mathrm{MPa}, 54.65 \mathrm{MPa}$ and $51.15 \mathrm{MPa}$. Statistical analysis showed that there was significantly related to the lateral stress.

Average tensile strength of bamboo with lateral stress of 0 $\mathrm{MPa}, 1.5 \mathrm{MPa}, 2 \mathrm{MPa}$ and 2.5 $\mathrm{MPa}$ were $132.54 \mathrm{MPa}, 146.69$ $\mathrm{MPa}, 122.01$ and $121.52 \mathrm{MPa}$. Statistical analysis showed the lateral stress of $2.5 \mathrm{MPa}$ was significantly.

\section{Acknowledgment}

The first outhor thanks to Structure Laboratory of Civil Department of Gadjah Mada University for research facilities. Authors to thank to Universitas Sarjanawiyata Tamansiswa (UST) for providing the research services.

\section{References}

[1] Morisco, Teknologi Bambu. Departement of Civil Engingeering and Enviromental, Gadjah Mada University, Yogyakarta, 2006.

[2] Amirullah, The influence of pressure on the shear strength of the beam clamp horizontally laminated bamboo Ampel. Departement of Civil Engingeering and Enviromental, Gadjah Mada University, Yogyakarta, 2007.

[3] Oka, I.M, Effect of pressure on the shear strength of the beam clamp horizontally laminated bamboo Petung. Faculty of Engineering, Gadjah Mada University, Yogyakarta, 2004

[4] Masrizal, The effect of compression force MoR Petung Bamboo laminated beams vertically. Departement of Civil Engingeering and Enviromental, Gadjah Mada University, Yogyakarta, 2004

[5] Ochi, Shinji, Tensile Properties of Bamboo Fiber Reinforced Biodegradable Plastics. International Journal of Composite Materials, 2(1) : 1-4., 2012. 